\title{
Differences in the Long-term Progression Course of Two Cases of Pellucid-like Keratoconus: Are they the Same Condition with Different Phenotypic Presentation?
}

\author{
Antonio Martínez-Abad ${ }^{1}$, David P Piñero ${ }^{2}$
}

\begin{abstract}
We report the long-term follow-up of two cases of untreated corneal ectasia presenting a crab claw-like sagittal and tangential topographic pattern at baseline, but without peripheral thinning. Currently, according to clinical examination, these two cases are diagnosed as pellucidlike keratoconus (PLK). The first case was a 58-year-old male who developed a true pellucid marginal degeneration (PMD) in one eye and with a nonprogressive PLK in the other eye. The second case presented was a 19-year-old male without clinical signs of ectasia at baseline that progressed to PLK with evident changes in topographic and pachymetric maps but maintaining the point of minimum corneal thickness (MCT) in the central area. The presence of two different conditions in the same patient (case 1) and the progression from inferosuperior asymmetry to the development of a crab claw-pattern (case 2) suggest that PMD, PLK, and keratoconus may be different phenotypic presentations of the same pathophysiological condition.
\end{abstract}

Keywords: Corneal ectasia, Keratoconus, Keratoconus progression, Pellucid marginal degeneration.

International Journal of Keratoconus and Ectatic Corneal Diseases (2019): 10.5005/jp-journals-10025-1176

\section{INTRODUCTION}

Pellucid marginal degeneration (PMD) is a noninflammatory and progressive ectatic corneal disease characterized by a narrow band of corneal thinning 1-2 $\mathrm{mm}$ from the limbus (commonly inferior limbus). ${ }^{1,2}$ It is considered as a rare condition ${ }^{1}$ and is usually diagnosed in the later decades of life compared to keratoconus. ${ }^{3}$ Furthermore, PMD seems to progress slower than keratoconus, presenting less visual alteration over time. ${ }^{4}$

Classic manifestation of PMD is flattening of the vertical meridian above the thinning band, inducing an area of steepening below this band, an "against-the-rule" astigmatism, ${ }^{5}$ and a crab claw-like topographic pattern. ${ }^{6}$ However, this specific topographic pattern may also be present in keratoconus, easily being misdiagnosed as a true PMD in clinical practice. ${ }^{7,8}$ Additional tools must be used for an adequate diagnosis of PMD, such as slit-lamp examination and pachymetric data, allowing the clinician to identify the position of the point of $\mathrm{MCT}^{2}$

Those cases presenting a crab claw-like pattern in the anterior corneal topographic map, but without associated peripheral thinning, cannot be diagnosed as true PMD. ${ }^{7,9}$ These cases have been defined in some series as "pellucid-like keratoconus" (PLK), being considered as a different presentation of keratoconus. Pellucid-like keratoconus differs clearly from the typical clinical manifestation of keratoconus, consisting on a well-delimited zone with a high dioptric value surrounded by zones of progressively decreasing corneal power combined with central or paracentral thinning. ${ }^{4,10}$ Therefore, the natural course of this type of keratoconus should be investigated and characterized in order to identify cases at risk and to define an accurate prognosis of each condition.

In this case report, we investigate and show the long-term course of two patients developing PLK, with similar clinical manifestations, but different age of presentation and level of ectasia progression over time.
${ }^{1}$ Department of Research and Development, Vissum Corporation, Alicante, Spain

${ }^{2}$ Department of Optics, Pharmacology and Anatomy, Group of Optics and Visual Perception, University of Alicante, Alicante, Spain

Corresponding Author: David P Piñero, Department of Optics, Pharmacology and Anatomy, Group of Optics and Visual Perception, University of Alicante, Alicante, Spain, Phone: +34-965903500, e-mail: david.pinyero@ua.es

How to cite this article: Martínez-Abad A, Piñero DP. Differences in the Long-term Progression Course of Two Cases of Pellucid-like Keratoconus: Are they the Same Condition with Different Phenotypic Presentation? Int J Kerat Ect Cor Dis 2019;8(1):29-33.

Source of support: The author David P Piñero has been supported by the Ministry of Economy, Industry and Competitiveness of Spain within the program Ramón y Cajal, RYC-2016-20471.

Conflict of interest: None

\section{Case Description}

\section{Case 1: Nonprogressive PLK}

A 58-year-old male attended to our ophthalmological center 12 years ago asking for a solution for the very advanced PMD that was present in his left eye (LE). This condition was treated with intrastromal corneal ring segment (ICRS) implantation in order to achieve a better control of corneal astigmatism and visual quality in this eye. In the right eye (RE), biomicroscopic signs compatible with corneal ectasia were not visible on slit-lamp examination, with an uncorrected visual acuity (UCVA) of 1.00 $\log M A R$, manifest refraction of $-3.00 \times 90^{\circ}$, and best corrected visual acuity (BCVA) of 0.0 logMAR. However, corneal topography revealed that at this baseline visit the presence of a band of maximum steepening in the inferior cornea and consequently

(O) The Author(s). 2019 Open Access This article is distributed under the terms of the Creative Commons Attribution 4.0 International License (https://creativecommons. org/licenses/by-nc/4.0/), which permits unrestricted use, distribution, and non-commercial reproduction in any medium, provided you give appropriate credit to the original author(s) and the source, provide a link to the Creative Commons license, and indicate if changes were made. The Creative Commons Public Domain Dedication waiver (http://creativecommons.org/publicdomain/zero/1.0/) applies to the data made available in this article, unless otherwise stated. 

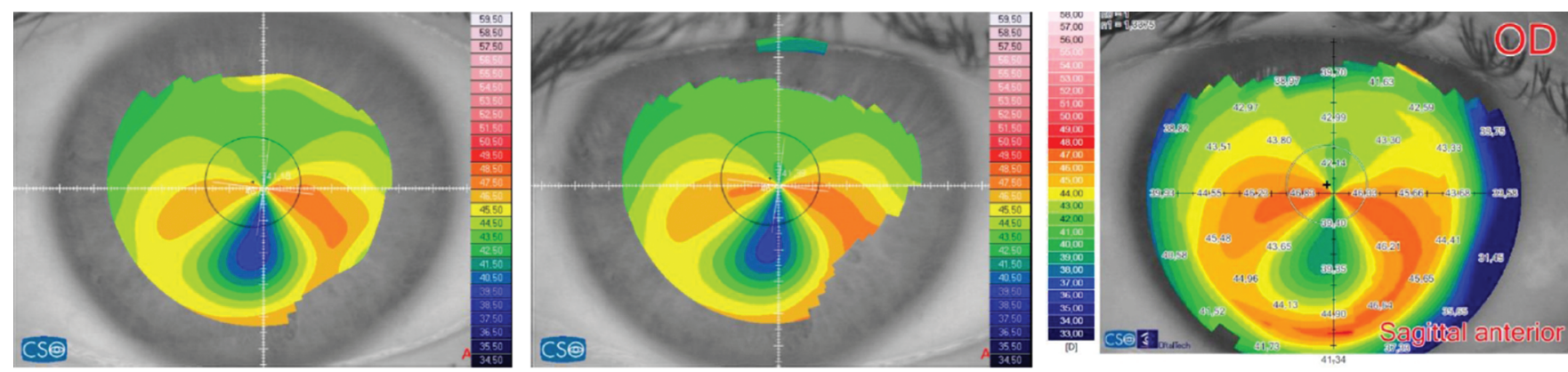

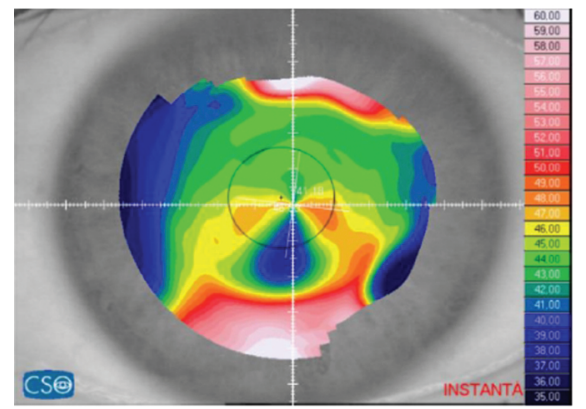

First examination

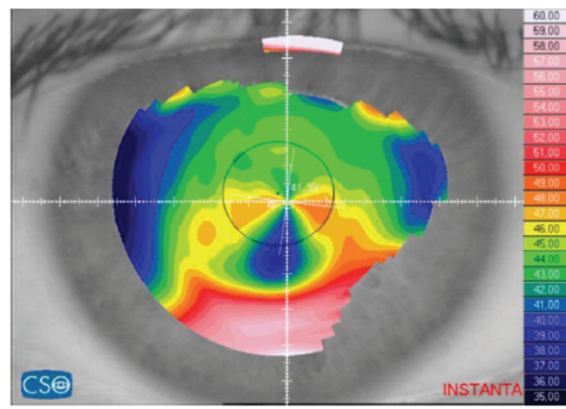

6 months
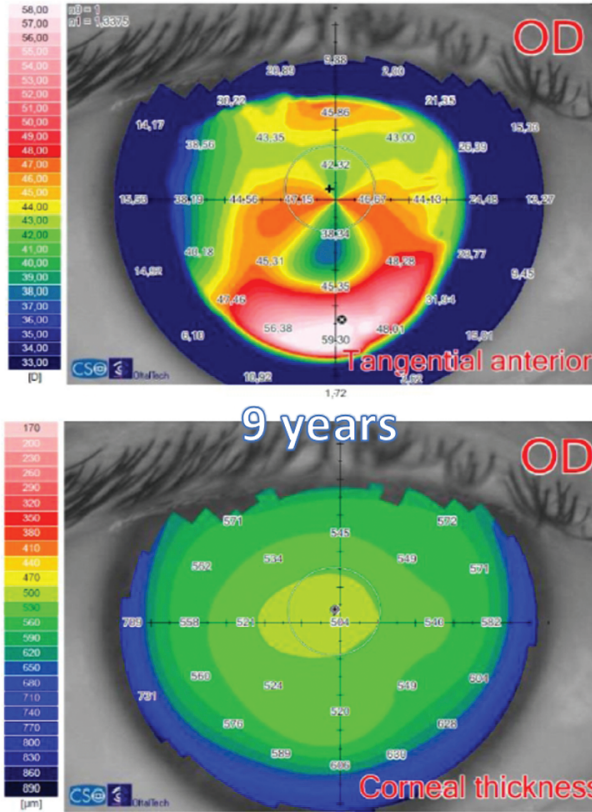

Fig. 1: Topographic maps of case 1, nonprogressive pellucid-like keratoconus: left-up, sagittal anterior map at first visit; left-center, tangential anterior map first visit; center-up, sagittal anterior map at 6 months after baseline examination; center-center, tangential anterior map at 6 months after baseline examination; right-up, sagittal anterior map at 9 years after baseline examination; right-center, tangential anterior map at 9 years after baseline examination; and right-down, corneal pachymetric map at 9 years after baseline examination

a flattening of the vertical meridian above the band, inducing a marked "against-the-rule" astigmatism. These morphological characteristics were associated with a crab claw-like topographic pattern (Fig. 1 left). At baseline, corneal topographic examination was performed with a CSO Eye Top system (Construzione Strumenti Oftalmici, Firenze, Italy), which is a Placido-based system evaluating the geometry of the anterior surface of the cornea. Furthermore, ultrasound pachymetry showed a baseline MCT of $485 \mu \mathrm{m}$. A consistent diagnosis of PMD was not possible considering that the peripheral band of thinning was absent. Periodic eye examinations including corneal topography was recommended for this eye.

Changes in topographic parameters during the follow-up are summarized in Table 1. Six months after the first examination, a complete eye examination was performed, obtaining an UCVA of $0.70 \log M A R$, manifest cylinder of $-4.00 \times 80^{\circ}$, and BCVA of 0.0 logMAR. Ectatic biomicroscopic signs were not appreciated, and topographic maps confirmed (Fig. 1 center) the absence of disease progression (Table 1). Corneal biomechanical response was analyzed in this visit with an Ocular Response Analyzer (ORA, Reichert Technologies, Buffalo, New York, USA) obtaining a corneal hysteresis $(\mathrm{CH})$ and corneal resistance factor (CRF) of 9.7 and $11.5 \mathrm{~mm} \mathrm{Hg}$, respectively.
Table 1: Keratometric and topographic parameters in the most relevant visits of case 1, nonprogressive pellucid-like keratoconus

\begin{tabular}{lccc}
\hline & First examination & After 6 months & After 9 years \\
\hline Sim K1 (D) & 41.18 & 41.39 & 41.04 \\
Sim K2 (D) & 46.80 & 46.95 & 46.76 \\
Sim Km (D) & 44.30 & 44.36 & 43.71 \\
Sim Ast (D) & -5.63 & -5.57 & -5.71 \\
$\mathrm{Q}_{4.5 \mathrm{~mm}}$ & 0.06 & 0.18 & 0.20 \\
$\mathrm{Q}_{8.0 \mathrm{~mm}}$ & -0.07 & -0.10 & 0.19 \\
SI & 0.02 & 0.52 & -0.96 \\
\hline
\end{tabular}

Sim, simulated; D, diopters; Q, asphericity; SI, symmetry index

Annual controls were recommended in this case in order to evaluate the course of the ectatic process. The patient attended to all planned visits, not obtaining refractive or topographic progression signs. The total follow-up period of this case to this date is 9 years. In his last visit, this eye was asymptomatic and did not show ectatic biomicroscopic signs, with a refraction of $+1.00-4.50 \times 85^{\circ}$ and a BCVA of $0.0 \log$ MAR. The crab claw-like topographic pattern was still present (Fig. 1 right), although a different topographic system was used (Sirius, Construzione 
Strumenti Oftalmici, Firenze, Italy). This system also provided a pachymetric map, showing a central location of the MCT point (Fig. 1 right-down), with no peripheral corneal thinning. This confirmed the diagnosis of PLK despite the presence of PMD in the other eye.

\section{Case 2: Progressive PLK}

A 19-year-old male attended to our ophthalmological center 12 years ago asking about refractive surgery and the possibility of achieving complete spectacle independence. He was a contact lens wearer, not referring any associated ocular and visual problem. Visual examination showed a high bilateral myopia: RE with $-9.50-1.00$ $\times 155^{\circ}$ and LE with $-6.50-1.50 \times 180^{\circ}$, achieving a BCVA of 0.20 and 0.10 logMAR, respectively. Some level of hyperemia was present in the tarsal conjunctiva on slit-lamp examination of both eyes as well as eyelid papillae and mild peripheral pannus. Ultrasound central pachymetry was $510 \mu \mathrm{m}$ in both eyes. The topographic analysis (CSO Eye Top system) showed a mild inferosuperior asymmetry (1.14 and $0.67 \mathrm{D}$ in RE and LE, respectively), with a significant steepening in the inferior cornea (Fig. 2 left). However, the rest of the exploration was normal and not consistent with the diagnosis of corneal ectasia according to the standard criteria. ${ }^{10}$ Therefore, refractive surgery was indicated, recommending phakic intraocular lens implantation in RE and Epi-LASIK in LE. Despite this recommendation, the patient preferred to wait without undergoing this surgery.

Nine years later, the patient attended again to our clinic, referring problems of tolerance of his soft contact lenses. Hyperemia and papillae were present again in the tarsal conjunctiva. In addition, a significant increase of refractive astigmatism was observed, especially in $\mathrm{RE}$, with a refractive error of $-5.50-7.50 \times 75^{\circ}$ and a BCVA of $0.20 \log$ MAR. Concerning the corneal topographic analysis, a crab claw-like pattern was found (Fig. 2 center), but without peripheral thinning in the pachymetric map (Fig. 2 center-down). According to this, PLK was diagnosed, recommending a discontinuation of contact lens wear, daily application of antihistaminic eye drops to minimize inflammatory signs, and a new ocular examination in 6 months.

In the next two eye examinations, no significant changes were observed in the corneal ectasia. However, 2 years later, the patient referred a visual loss in his RE. In the visual examination, both sphere and cylinder were found to be significantly increased: a manifest refraction of $-8.00-8.75 \times 70^{\circ}$ and a BCVA of $0.20 \log M A R$. CH and CRF were also found to be reduced, at 7.4 and $7.0 \mathrm{~mm} \mathrm{Hg}$ in $\mathrm{RE}$ and $\mathrm{LE}$, respectively. Moreover, topographic maps presented changes indicating progression of the disease (Fig. 2 right and Table 2). CXL was then recommended by the ophthalmologist to halt the
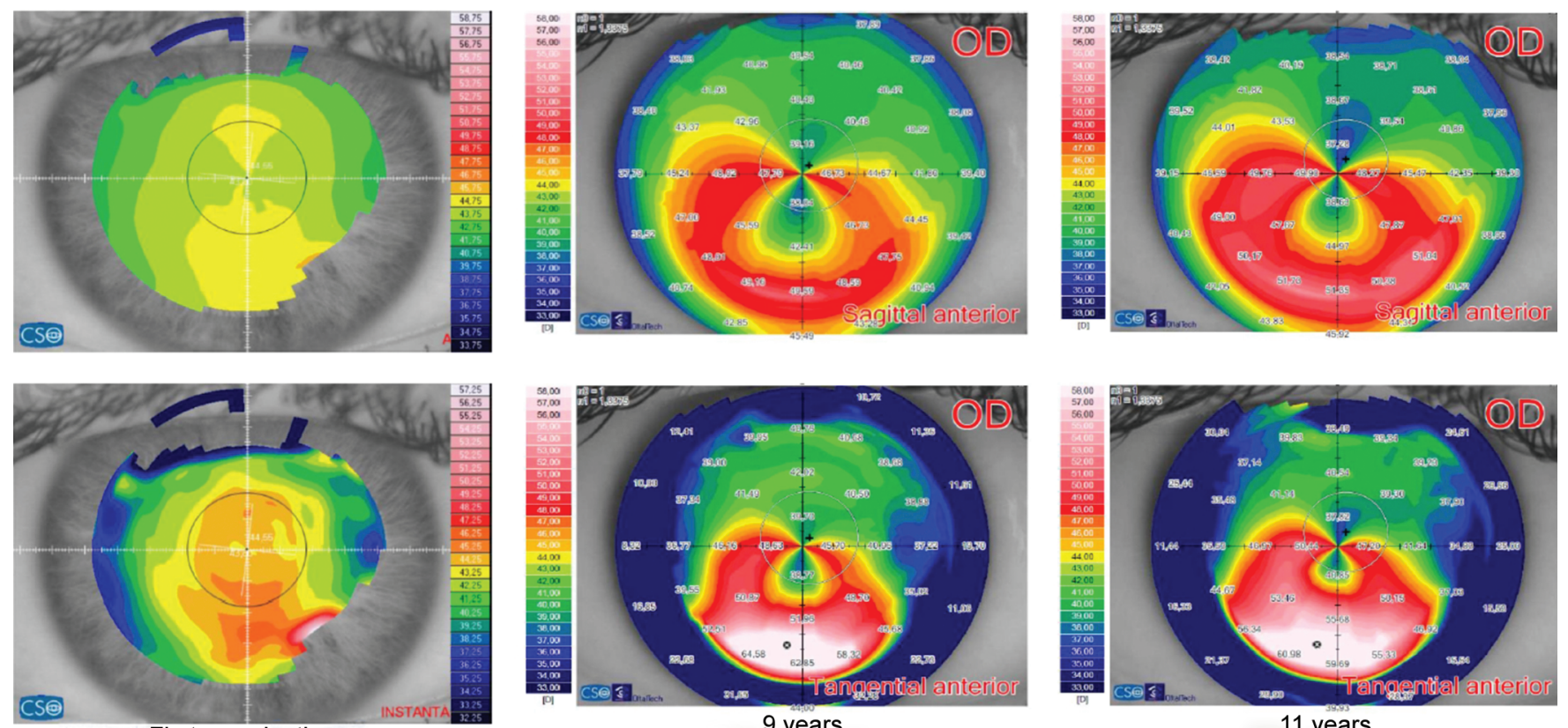

9 years
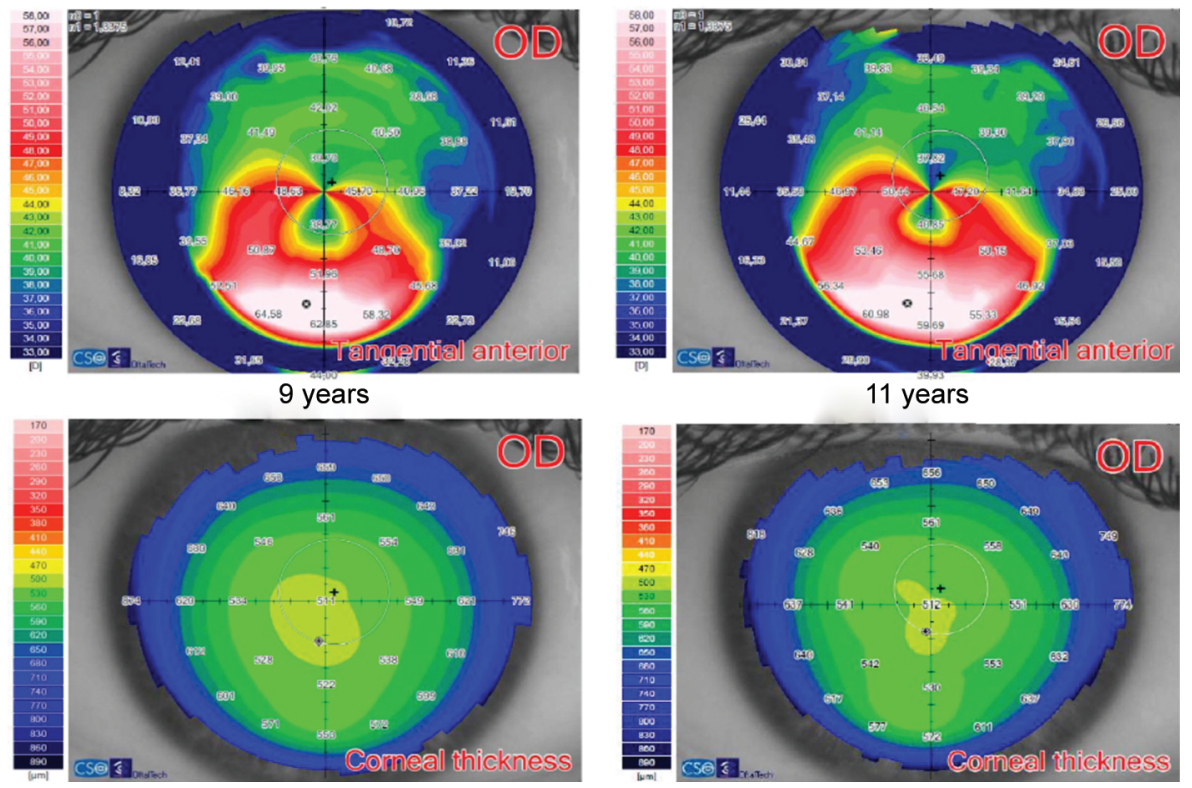

11 years

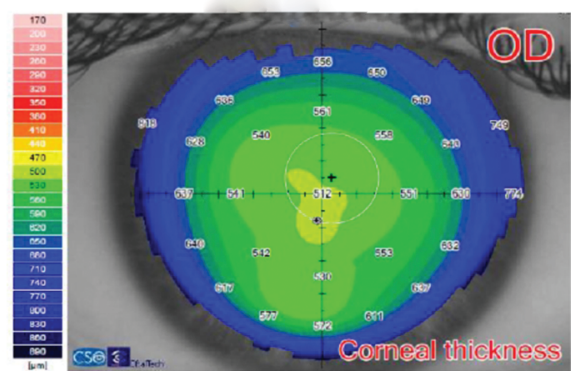

Fig. 2: Topographic maps of case 2, progressive pellucid-like keratoconus: left-up, sagittal anterior map at first visit; left-center, tangential anterior map at first visit; center-up, sagittal anterior map at 9 years after baseline examination; center-center, tangential anterior map at 9 years after baseline examination; center-down, corneal pachymetric map at 9 years after baseline examination; right-up, sagittal anterior map at 11 years after baseline examination; right-center, tangential anterior map at 11 years after baseline examination; and right-down, pachymetric map at 11 years after baseline examination 
Table 2: Keratometric and topographic parameters in the most relevant visits of case 2, nonprogressive pellucid-like keratoconus

\begin{tabular}{lccc}
\hline & First examination & After 6 months & After 9 years \\
\hline Sim K1 (D) & 43.80 & 39.53 & 39.19 \\
Sim K2 (D) & 44.55 & 47.48 & 48.91 \\
Sim Km (D) & 44.17 & 43.14 & 43.51 \\
Sim Ast (D) & -0.74 & -7.95 & -9.71 \\
Q $_{4.5 m m}$ & 0.11 & 0.73 & 0.81 \\
Q $_{8.0 m m}$ & -0.14 & 0.34 & 0.34 \\
SI & 1.15 & 3.06 & 5.72 \\
\hline
\end{tabular}

Sim, simulated; D, diopters; $Q$, asphericity; Sl, symmetry index

progression of the disease, with implantation of ICRS afterward to reduce corneal astigmatism and irregularity.

\section{Discussion}

Differential diagnosis between all corneal ectatic disorders is not always an easy task. The absence of inflammatory signs and the close similarities between some of them, especially in atypical manifestations of the diseases, make a consistent diagnosis difficult. The typical clinical manifestation of keratoconus is the presence of a conical protrusion involving the central or paracentral cornea combined with central thinning. ${ }^{4,10}$ In contrast, PMD is commonly characterized by a peripheral crab claw-like steepening combined with peripheral thinning; ${ }^{1,2}$ keratoglobus, which is the least common of all corneal ectatic disorders, is characterized an entire cornea being affected. ${ }^{11}$ In the past, when corneal topographers only provided data of the anterior corneal surface, the corneal thinning had to be identified on slit-lamp examination. This is extremely complicated in early stages, and clinicians used to base their diagnosis on the anterior corneal topographic pattern, being considered as the main tool to distinguish between keratoconus and PMD. However, the development of Scheimpflug imaging-based devices and optical coherence tomography (OCT) technology in the past years has allowed the clinician to obtain an analysis of both anterior and posterior corneal surfaces and consequently pachymetric maps. With all this information, it has been demonstrated that some keratoconus cases with a crab claw-like pattern can be misdiagnosed as PMD. ${ }^{5,7,9}$ Sinjab and Youssef ${ }^{7}$ analyzed the tomographic features in corneas with the crab claw-like topographic pattern, demonstrating that only $26.7 \%$ of cases had a real PMD and the rest $(73.7 \%)$ had PLK. Similarly, Lee et al. ${ }^{5}$ performed a similar study in a larger sample, obtaining equivalent results (23.7\% PMD vs 71.1\% PLK). Fuchihata and Maeda ${ }^{9}$ analyzed the appearance of the topographic pattern in true PMD (confirmed by pachymetric map), not observing a crab claw-like pattern in any case (only 78\%).

In the two cases reported, a crab claw-like pattern was observed in the sagittal and tangential maps at least in one of the examinations (case 1, Fig. 1; case 2, Fig. 2), with no peripheral thinning in the pachymetric map. For this reason, both cases were diagnosed as PLK. However, the evolution of each case was completely different: case 1 was compatible with PLK in one eye and true PMD in the fellow eye at the first examination, with no significant changes in terms of refraction, topography, pachymetry, and biomicroscopic findings during a 9-year follow-up. He was 58 years old and the prognosis differs from that reported for some PMD cases showing changes at older ages. ${ }^{12}$ This brought up several questions: how was the morphological process of development of this kind of ectasia? When and how did the clinical manifestations start?
These questions can be extrapolated to case 2. In this case, a 30-year-old man developed an ectasia with manifestation of PLK during a 11-year follow-up. Therefore, PLK cases can be also progressive, even between the third and the fourth decade of life. It should be considered that a crab claw-like topographic pattern was absent at baseline (only inferosuperior asymmetry compatible with subclinical keratoconus (Fig. 2 left)). Nine years after baseline examination, the corneal disorder progressed completely changing the topographical shape of the cornea (well-delimited crab claw-like protrusion). Concerning pachymetric data, no peripheral thinning was present during the first period of the follow-up, with a trend to a thinning in the inferior cornea in the last tomographic examination. However, this trend was not enough to provide a consistent diagnosis of PMD. This brings up the following question: is this condition a first stage of progression of true PMD? This question is not admissible as CXL was required to be applied as proofs of ectasia progression were detected. However, the displacement of the maximum thinning to an inferior area suggests this potential progression.

The potential presence of PMD in a case where previously a PLK was present (case 2 ) in combination with the presence of PLK in one eye and true PMD in the fellow eye of the same patient (case 1) suggest that PMD and keratoconus may represent the same underlying disorder with different clinical manifestations. Particularly, PMD may be a peripheral form of keratoconus, as suggested previously by several authors. ${ }^{7,9}$ Therefore, PLK and PMD would represent the same disorder but at different stages, PMD being an advanced type of manifestation of the ectatic disorder. In agreement with this hypothesis, Karabatsas and Cook ${ }^{13}$ revealed associations between PMD and keratoglobus, supporting the idea that all noninflammatory corneal disorders may be one continuous disorder with different phenotypes. To this date, histopathological studies have evaluated the biochemical differences in 11 tear mediators between PMD and keratoconus, presenting relevant variances only in one mediator (matrix metalloproteinases-9). It involves a very close origin between both processes, but more studies of biomarkers in different corneal ectatic presentations are required to confirm the presence of the only underlying disease.

In conclusion, the two cases reported are additional evidence supporting the hypothesis of a same pathophysiological condition in corneal ectasia, but with different phenotypic presentations. Future clinical and experimental studies should elucidate if the suggested hypothesis according to our evidence and previously reported proofs is correct.

\section{Disclosure}

The authors have no proprietary or commercial interest in the medical devices that are involved in this manuscript. David $P$ Piñero has been supported by the Ministry of Economy, Industry and Competitiveness of Spain within the program Ramón y Cajal, RYC-2016-20471.

\section{References}

1. Jinabhai $A$, Radhakrishnan $\mathrm{H}, \mathrm{O}^{\prime}$ Donnell C. Pellucid corneal marginal degeneration: a review. Cont Lens Anterior Eye 2011;34(2):56-63. DOI: 10.1016/j.clae.2010.11.007.

2. Martínez-Abad A, Piñero DP. Pellucid marginal degeneration: detection, discrimination from other corneal ectatic disorders and progression. Cont Lens Anterior Eye 2019;42(4):341-349. DOI: 10.1016/j.clae.2018.11.010.

3. Imbornoni LM, McGhee CNJ, Belin MW. Evolution of keratoconus: from diagnosis to therapeutics. Klin Monbl Augenheilkd 2018;235(6): 680-688. DOI: 10.1055/s-0044-100617. 
4. Martínez-Abad A, Piñero DP. New perspectives on the detection and progression of keratoconus. J Cataract Refract Surg 2017;43(9): 1213-1227. DOI: 10.1016/j.jcrs.2017.07.021.

5. Lee BW, Jurkunas UV, Harissi-dagher M, et al. Ectatic disorders associated with a claw-shaped pattern on corneal topography. Am J Ophthalmol 2007;144(1):154-156. DOI: 10.1016/j.ajo.2007.02.032.

6. Koc M, Tekin K, Inanc M, et al. Crab claw pattern on corneal topography: pellucid marginal degeneration or inferior keratoconus? Eye (Lond) 2018;32(1):11-18. DOI: 10.1038/eye.2017.198.

7. Sinjab MM, Youssef LN. Pellucid-like keratoconus. F1000Res 2012;1:48. DOI: 10.12688/f1000research.1-48.v1.

8. Tummanapalli SS, Maseedupally V, Optom BS. Evaluation of corneal elevation and thickness indices in pellucid marginal degeneration and keratoconus. J Cataract Refract Surg 2013;39(1):56-65. DOI: 10.1016/j.jcrs.2012.08.053
9. Fuchihata M, Maeda N. Characteristics of corneal topographic and pachymetric patterns in patients with pellucid marginal corneal degeneration. Jpn J Ophthalmol 2014;58(2):131-138. DOI: 10.1007/ s10384-013-0291-3.

10. Piñero DP, Nieto JC, Lopez-Miguel A. Characterization of corneal structure in keratoconus. J Cataract Refract Surg 2012;38(12): 2167-2183. DOI: 10.1016/j.jcrs.2012.10.022.

11. Wallang BS, Das S. Keratoglobus. Eye (Lond) 2013;27(9):1004-1012. DOI: 10.1038/eye.2013.130.

12. Bower KS, Dhaliwal DK, Barnhorst DAJ, et al. Pellucid marginal degeneration with superior corneal thinning. Cornea 1997;16(4): 483-485. DOI: 10.1097/00003226-199707000-00018.

13. Karabatsas $\mathrm{CH}$, Cook SD. Topographic analysis in pellucid marginal degeneration and keratoglobus. Eye (Lond) 1996;10(Pt 4):451-455. DOI: 10.1038/eye.1996.99. 